\title{
A new nonocclusive laser-assisted coronary anastomotic connector in a rabbit model
}

\author{
David Stecher, MD, ${ }^{\mathrm{a}}$ Bart de Boer, MD, ${ }^{\mathrm{b}}$ Cornelis A. F. Tulleken, MD, PhD, ${ }^{\mathrm{b}}$ Gerard Pasterkamp, MD, \\ $\mathrm{PhD},{ }^{\mathrm{c}}$ Lex A. van Herwerden, $\mathrm{MD}, \mathrm{PhD},{ }^{\mathrm{a}}$ and Marc P. Buijsrogge, $\mathrm{MD}, \mathrm{PhD}{ }^{\mathrm{a}}$
}

\begin{abstract}
Objective: The Excimer laser-assisted nonocclusive anastomotic technique is a nonocclusive, facilitated bypass technique that is currently Conformité Européenne and Food and Drug Administration approved for clinical application in neurosurgery. In the present study, we assessed the safety and feasibility of a newly developed Excimer laser-assisted nonocclusive anastomosis-based prototype coronary anastomotic connector in an acute rabbit abdominal aortic bypass model before application in experimental coronary bypass surgery. In addition, 2 sealants were tested to facilitate anastomotic hemostasis in the current device prototype.
\end{abstract}

Methods: A total of 40 anastomoses were constructed on the abdominal aorta (3.5 mm outer diameter) of 10 rabbits. The anastomotic circumference was sealed by a surgical sealant to obtain complete hemostasis (BioGlue vs TachoSil). The anastomoses were evaluated by flow measurements construction time, hemostasis, histologic analysis, and burst pressure testing.

Results: The connector enabled a nonocclusive and fast ( $6.0 \pm 1.7$ minutes, mean \pm SD [including sealing]) anastomosis construction and complete hemostasis in 95\% (35/37). Sealing with BioGlue was faster than with TachoSil (19\% vs 53\% of construction time). Despite technical imperfections ( $7 / 40$ failures to completely retrieve the flap by the laser), all 40 anastomoses were patent, showed reproducible construction with intimaadventitia apposition, streamlining thrombus coverage of the intraluminal laser rim, and no vessel wall damage. All anastomoses resisted ex vivo supraphysiologic pressures $(>300 \mathrm{~mm} \mathrm{Hg}$ ).

Conclusions: The results of the present study have demonstrated that the Excimer laser-assisted nonocclusive anastomotic connector is safe and reliable and can be efficiently applied in an acute rabbit abdominal aortic bypass model. Provided the limitations can be addressed, this easy-to-use and nonocclusive technique has the potential to facilitate minimally invasive coronary bypass surgery. (J Thorac Cardiovasc Surg 2013;145:1124-9)

To facilitate minimal access coronary artery bypass grafting, a reliable and simplified alternative for hand-sutured coronary anastomosis must be developed. The Excimer laser-assisted nonocclusive anastomotic (ELANA) technique $^{1}$ is a nonocclusive, facilitated bypass technique that is currently Conformité Européenne and Food and Drug Administration approved for clinical application in neurosurgery. In the present study, we evaluated the safety and feasibility of a newly developed ELANA-based prototype coronary anastomotic connector in an acute rabbit abdominal aortic bypass model before application in experimental

From the Department of Cardiothoracic Surgery ${ }^{\mathrm{a}}$ and Experimental Cardiology ${ }^{\mathrm{c}}$ of the Heart and Lungs Division, and Department of Neurosurgery of the Brain Division, ${ }^{\mathrm{b}}$ University Medical Center Utrecht, Utrecht, The Netherlands.

This study was supported by the University Medical Center Utrecht, ELANA b.v. and Eurostars EUREKA, project NewHeart (ESTAR10210).

Disclosures: C.A.F. Tulleken holds shares in ELANA b.v., Utrecht, The Netherlands. The other authors have nothing to disclose with regard to commercial support.

Received for publication March 27, 2012; revisions received May 7, 2012; accepted for publication May 17, 2012; available ahead of print June 28, 2012.

Address for reprints: David Stecher, MD, Department of Cardiothoracic Surgery, University Medical Center Utrecht, Room E03.511, PO Box 85500, Utrecht, 3508 GA, The Netherlands (E-mail: dsteche2@umcutrecht.nl).

$0022-5223 / \$ 36.00$

Copyright (c) 2013 by The American Association for Thoracic Surgery doi:10.1016/j.jtcvs.2012.05.061 coronary bypass surgery. In addition, 2 types of sealant were tested (BioGlue vs TachoSil) to facilitate optimal anastomotic hemostasis in the current device prototype.

\section{METHODS \\ ELANA Prototype Anastomotic Connector and Procedure}

The ELANA anastomotic connector consists of a 2.6-mm (inner diameter) stainless steel ring (thickness $0.25 \mathrm{~mm}$ ) with 2 pins $(6.2 \mathrm{~mm}$ length; Figure 1, A). The current prototype is suitable for target vessels with a 3- to 5-mm outer diameter (OD) in either an end-to-side or a sideto-side configuration. The bypass graft (5-6 mm OD) is currently mounted onto the connector (arteriotomy, $2.5 \mathrm{~mm}$ long) using 6 Prolene 8-0 stitches (Ethicon, Somerville, NJ) such that the intima of the graft completely covered the inner surface of the ring (Figure 2, A). The mounted ELANA connector is inserted into the recipient artery by the introduction of the 2 pins using a standard needle holder (spring-style needle holder; Scanlan International, Saint Paul, Minn) before the lasered arteriotomy. The pins puncture the recipient arterial wall, and the device is pushed forward (Figure 2, $B$ ). Subsequently, the pins penetrate outward again, and the connector is pushed further until the recipient arterial wall "clicks" over the circumferential part of the pins of the device (Figure 2, $C$ ). If proper positioning is established, the anastomotic circumference, in the current prototype, is sealed with a surgical sealant to achieve complete hemostasis. After polymerization of the sealant, the anastomotic site and donor are rinsed intraluminally with heparinized saline to prevent hindering of the laser-totissue contact by blood. Subsequently, a laser catheter $(2.5 \mathrm{~mm}$ OD; laser fibers, $2.0 \mathrm{~mm}$ OD; Figure 1, B) is introduced through the laser 


\section{Abbreviations and Acronyms \\ BENIS = blood-exposed nonintimal surface \\ ELANA $=$ Excimer laser-assisted nonocclusive anastomosis \\ OD $\quad=$ outer diameter}

introduction-conduit (ie, the free distal—not blood exposed —end of the graft) and perpendicularly positioned into the ring of the mounted device onto the recipient artery vessel wall (Figure 2,D). Vacuum suction through the lumen of the catheter is initiated onto the recipient artery wall, and the anastomosis is lasered open, without occluding the recipient artery, resulting in an anastomotic orifice with a 2-mm diameter. Subsequently, the laser catheter is retrieved, including the lasered fragment of the arterial wall ("flap") attached to the grid of the vacuum lumen (Figure 2, E). A small protruding adventitial rim of the abdominal aorta, the laser edge, is exposed intraluminally after lasering the anastomosis (Figure 2, E, magnified section). To complete the anastomosis, the distal end (laser introduction conduit or "cul de sac") of the graft is ligated using a hemoclip (Weck Hemoclip; Teleflex Medical, Research Triangle Park, NC). After construction of the second anastomosis, the flow over the bypass is initiated (Figure 2, F).

\section{Rabbits}

Ten New Zealand white rabbits $(3 \mathrm{~kg}$ ) were used. The rabbits were fed a normal diet and received humane care in compliance with the "Guide for the Care and Use of Laboratory Animals" (Institute of Laboratory Animal Resources, National Research Council [revised 1996]). The animal experimentation committee of the Utrecht University approved the protocol. The rabbits did not receive any anticoagulant or antiplatelet medication preoperatively.

\section{Anesthesia and Euthanasia}

Anesthesia was induced with acepromazine $(0.5 \mathrm{mg} / \mathrm{kg})$, methadone $(1 \mathrm{mg} / \mathrm{kg})$ intramuscularly and etomidate $(0.3 \mathrm{mg} / \mathrm{kg})$ intravenously. After intubation and ventilation, anesthesia was maintained by continuous intravenous infusion of midazolam (50 $\mu \mathrm{g} / \mathrm{h} / \mathrm{kg})$ and sufentanil $(50 \mu \mathrm{g} / \mathrm{h} / \mathrm{kg})$. The rabbit was put in the supine position, and mean arterial blood pressure was kept at $60 \mathrm{~mm} \mathrm{Hg}$ during the whole procedure. The rabbits were killed with $200 \mathrm{mg} / \mathrm{kg}$ sodium pentobarbital intravenously.

\section{Surgery and Experimental Model}

The abdominal aorta ( $3.5 \mathrm{~mm}$ OD) was dissected from its bifurcation up to the left renal artery using a microscope (Leica MS1; Leica Microsystems $\mathrm{GmbH}$, Wetzlar, Germany), and the loose periadventitial tissue was removed. Before anastomosis construction, heparin was administered intravenously to obtain an activated clotting time (Hemotec, Englewood, Colo) of at least 4 times the normal value. Two bypass constructions, each consisting of 2 side-to-side anastomoses (Figure 2, F), were constructed on the abdominal aorta using an ex vivo rabbit thoracic aorta bypass graft (5-6 mm OD; total 10 rabbits, 40 anastomoses). In the present study, 2 different sealants were evaluated to seal the anastomosis: BioGlue (Cryolife, Kennesaw, Ga) and TachoSil (Nycomed, Zurich, Switzerland). After construction of 2 anastomoses, the native abdominal aorta was occluded in between the anastomoses using an aneurysm clip (Yasargil aneurysm-clip system; Peter Lazic, GmbH, Tuttilingen, Germany) after flow over the bypass was initiated.

The safety and feasibility of the anastomotic technique were assessed (in a nonblinded fashion by either 1 or 2 independent investigators) by the anastomotic construction time (assessed by 1 investigator), patency (ie, flow measurements and histologic examination), vessel wall apposition, thrombus formation, and vessel wall damage. Furthermore, the reliability of 2 surgical sealants was assessed by the application time (assessed by 1 investigator), hemostasis, sealant localization (histologic examination), any potential acute inflammatory cell reactions, and burst pressure testing. The anastomoses $(n=40)$ were evaluated intraoperatively, at 1 hour, and after death.

\section{Flow Measurements}

The baseline abdominal aortic flow $(\mathrm{mL} / \mathrm{min})$ was measured with a calibrated transit time flow probe (3S model T208; Transonic Systems, Ithaca, NY) before anastomosis construction at a mean blood pressure of $60 \mathrm{~mm} \mathrm{Hg}$. After anastomosis construction, aortic flow was continuously measured and recorded for at least 1 hour at $60 \mathrm{~mm} \mathrm{Hg}$ until termination.

\section{Hemostasis}

After anastomotic construction, leakage was assessed. Hemostasis was quantified as completely hemostatic, oozing, or brisk leakage. Any leakage that was self-limiting or could be controlled by short tamponation (maximally 3 minutes) was defined as oozing. If it was necessary to add more sealant, a stitch was necessary, or the artery had to be occluded temporarily to obtain hemostasis, the leakage was defined as brisk.

\section{Histologic Examination}

After death, the anastomoses were removed and flushed under low pressure with heparinized saline. From each bypass, 1 anastomosis was fixated overnight in $4 \%$ formalin for histologic analysis $(n=20)$. The anastomoses were embedded in plastic (methyl methacrylate) and subsequently sectioned in transverse $(n=10)$ or longitudinal $(n=10)$ planes (at $300-\mu \mathrm{m}$ intervals), starting at $5 \mathrm{~mm}$ downstream and continuing up to $5 \mathrm{~mm}$ upstream of the anastomosis. The sections were stained with hematoxylin and eosin. Vessel wall apposition (eg, intima-adventitia, intima-intima), protrusion of the intraluminal rim (millimeters), thrombus formation at the intraluminal rim (height of thrombus [micrometers]), and bloodexposed nonintimal surface ${ }^{2}$ (BENIS; medial and adventitial surface [intraluminal exposed rim] and intraluminal connector surface area) were recorded. The tissue damage (eg, medial necrosis of recipient vessel; ie, opposite and lateral vessel walls and intraluminal vessel wall rim [pyknosis of smooth muscle cell nuclei]), acute inflammatory cell reaction (polymorphonuclear cells and macrophages) of the recipient vessel wall, and sealant localization (eg, inside and outside the anastomosis) were assessed. Measurements were performed using the software package AnalySiS (Soft-Imaging Software GmbH, Münster, Germany).

\section{Burst Pressure}

Of each bypass, 1 anastomosis was used for burst pressure testing $(\mathrm{n}=20)$. The anastomoses that were intraoperatively classified as oozing or completely hemostatic were included in the burst pressure analysis. To test the burst pressure, the anastomoses were attached to a calibrated pulsatile pressure system with water. The pressure was increased by 100 $\mathrm{mm} \mathrm{Hg} / \mathrm{min}$ until a maximum of $300 \mathrm{~mm} \mathrm{Hg}$ was reached. If detachment occurred and/or leakage appeared at the anastomotic line, the anastomosis was scored as burst and the pressure recorded.

\section{Statistical Analysis}

The data are presented as the mean \pm standard deviation or as noted otherwise. To assess the differences in sealing time between the 2 subgroups, the $P$ values were calculated using the Mann-Whitney $U$ test (SPSS version 17.0; SPSS, Chicago, Ill).

The Wilcoxon signed ranks test was used to assess differences in aortic flow before and after anastomosis construction (SPSS version 17.0). 

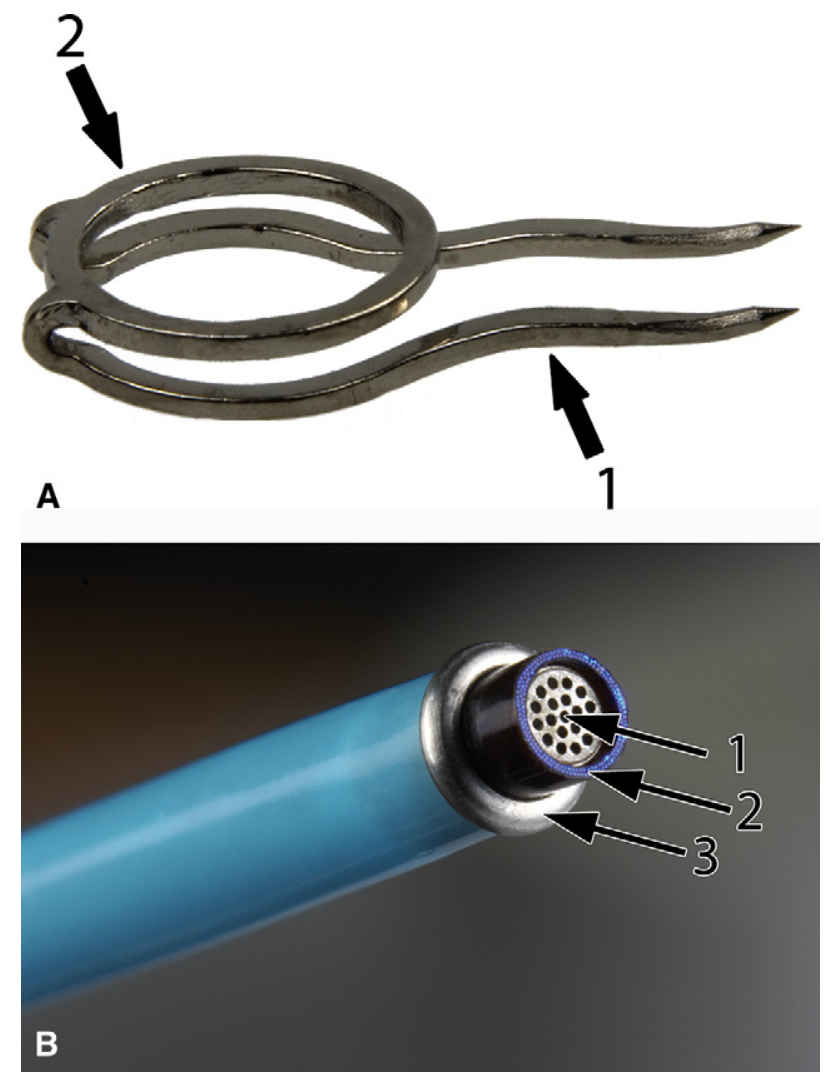

FIGURE 1. Excimer laser-assisted nonocclusive anastomosis prototype anastomotic connector and laser catheter. A, Prototype anastomotic connector with 2 sharp pins (1) (6.2 mm length) attached to a ring (2) (2.6 $\mathrm{mm}$ inner diameter). B, Laser catheter ( $2.5 \mathrm{~mm}$ outer diameter): the grid of the vacuum channel (1) is located centrally and surrounded by laser fibers (2) (2.0 mm outer diameter). The outer band (3) (3.0 mm outer diameter) provides safety and stabilization into the ring of the anastomotic connector.

\section{RESULTS \\ Surgery}

All anastomoses were performed by 1 investigator (D.S.). The mean anastomosis construction time was $6.0 \pm 1.7$ minutes (Table 1), of which an average of $2.3 \pm 1.6$ minutes ( $38 \%$ of construction time) was required to seal the anastomosis. Sealing with BioGlue was faster than with TachoSil $(19 \%$ vs $53 \%$ of construction time, $P<.01$; Table 1$)$. Mounting of the graft onto the prototype connector required $20.3 \pm 3.2$ minutes. Other operative data are listed in Table 2. After lasering the anastomosis, a flap was successfully retrieved in $33(83 \%)$ of 40 anastomoses (Table 2).

\section{Flow Measurements}

All individual anastomoses $(n=40)$ showed consistent graft flow until termination. The mean aortic flow after construction of all the anastomoses $(\mathrm{n}=4$ per aorta) was lower than the baseline aortic flow $(45 \pm 18 \mathrm{~mL} / \mathrm{min}$ vs $72 \pm 28$ $\mathrm{mL} / \mathrm{min}, P<.01)$.

\section{Hemostasis}

Of all the anastomoses, $95 \%(35 / 37)$ were completely hemostatic after construction (Table 2), and in 5\% (2/37) brisk leakage occurred. In the latter 2 cases (TachoSil subgroup), the leakage was caused by insufficient application or detachment of the sealant. In both cases, reapplication of the sealant resulted in complete hemostasis. Complete direct hemostasis was achieved in all anastomoses of the BioGlue subgroup.

\section{Histologic Examination}

Vessel wall apposition. In all anastomoses, the intima of the graft was opposed to the adventitia of the recipient aorta along the full circumference of the anastomosis, and a small protruding rim of the recipient artery (mean length, $0.11 \pm 0.12 \mathrm{~mm}$ ) was exposed intraluminally (Figure 3). A sharp laser-cut edge with a streamlined cover of platelets and fibrin (mean height, $37 \pm 21 \mu \mathrm{m}$ ) was observed. No platelets or fibrin were seen at the intraluminal exposed part of the connector. In cases in which the flap was not retrieved directly by the catheter, an incompletely lasered flap resided in the anastomotic orifice partly attached by a small, nonlasered bridge of aortic vessel wall. No evidence of distal embolization of parts of the flap was found. BENIS. The anastomoses showed a BENIS area of approximately $4.4 \mathrm{~mm}^{2}$ (intraluminal connector surface area, $\sim 2.5$ $\mathrm{mm}^{2}$ and intraluminal exposed rim, $\sim 1.9 \mathrm{~mm}^{2}$ ).

Medial necrosis. No medial necrosis was observed near the connector or sealant. In addition, at the intraluminal rim, we did not observe medial cell damage caused by the laser, but a sharp cut edge of viable rim of the recipient vessel wall. No medial necrosis was observed at the recipient arterial wall. However, compression of the recipient vessel wall was seen between the ring and the pins of the device. Sealant localization. Intraluminal exposure of sealant was seen in 1 anastomosis in the BioGlue subgroup and 1 in the TachoSil subgroup. No acute inflammatory cell reaction was found.

\section{Burst Pressure}

All anastomoses resisted a pressure of $300 \mathrm{~mm} \mathrm{Hg}$ (Table 2).

\section{DISCUSSION}

The principal results of the present study were as follows. First, despite technical imperfections (ie, failure to retrieve the flap by the laser catheter), all 40 anastomoses were patent and showed a predictable and consistent construction with intima-adventitia apposition in a rabbit abdominal aortic bypass model. Second, the technique enabled easy-to-use, nonocclusive, and fast (mean, $6.0 \pm 1.7$ minutes) anastomosis construction, and the anastomosis resisted ex vivo supraphysiologic pressures. Finally, the 


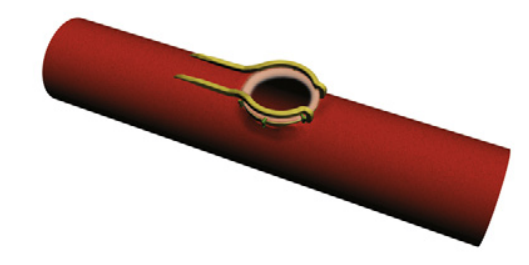

A

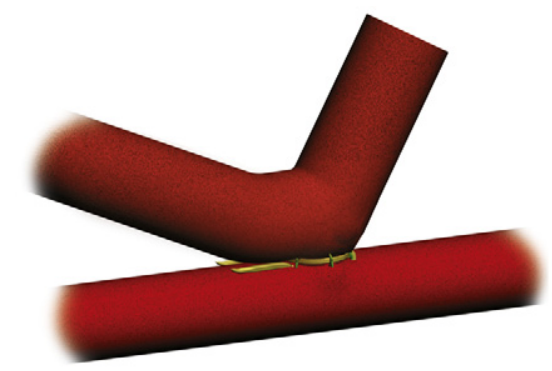

C

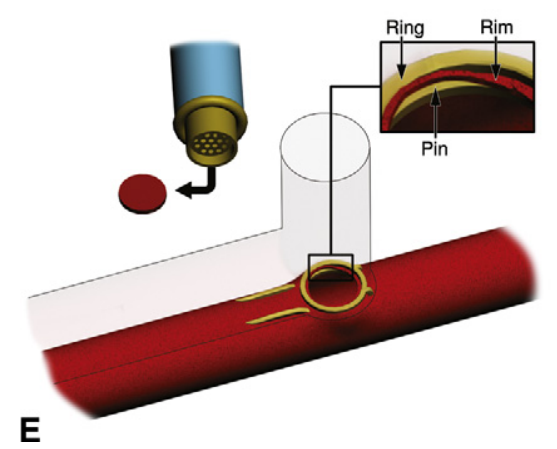

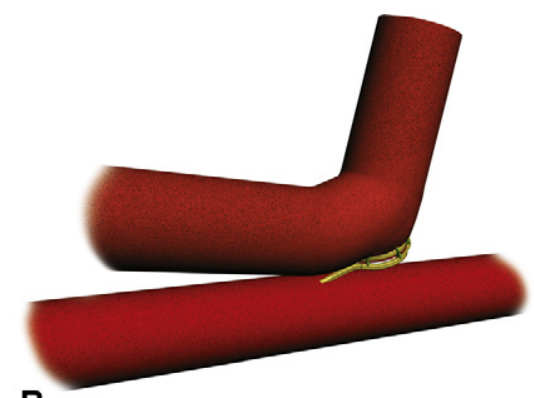

B

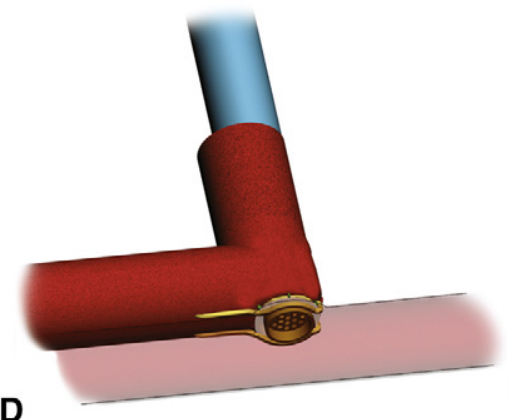

D

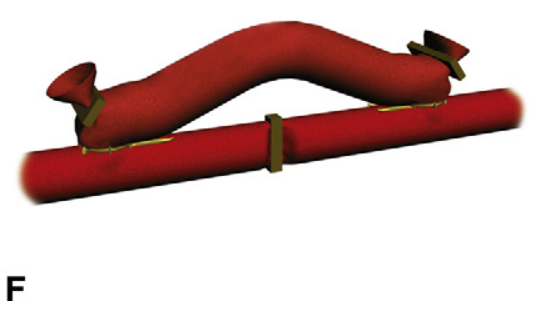

FIGURE 2. Excimer laser-assisted nonocclusive anastomotic procedure in the rabbit abdominal aortic bypass model. A, The graft is mounted onto the connector using 6 stitches. The intima of the graft (highlighted) completely covers the inner surface of the ring of the connector. B, A standard needle holder (not shown) is used to insert the connector. The pins of the connector puncture the abdominal aortic wall. C, The pins penetrate outward again. The anastomotic circumference is sealed by a surgical sealant (not shown). D, Perpendicular positioning of the laser catheter (intima of the graft is highlighted). E, The catheter with a lasered fragment ("flap") of the abdominal aortic wall (graft illustrated transparently). A small protruding adventitial rim of the abdominal aorta, the laser edge, is exposed intraluminally (see magnified section [complete coverage of the ring of the connector by the graft not shown]). F, Final abdominal aortic bypass with 2 anastomoses. Ligating hemoclips are placed at both ends of the graft, and a clip occludes the abdominal aorta in between the 2 anastomoses.

anastomoses sealed with BioGlue demonstrated more favorable results with regard to construction time and hemostasis.

TABLE 1. Anastomotic construction and sealing time

$\left.\begin{array}{lcc}\hline & \text { Construction time }(\mathbf{m i n}) & \text { Sealing time }(\mathbf{m i n}) \\ \hline \text { Total } & 6.0 \pm 1.7 & 2.3 \pm 1.6(38 \% *) \\ \text { BioGlue } & 4.5 \pm 0.5 & 0.9 \pm 0.4(19 \% *) \\ \text { TachoSil } & 7.3 \pm 0.8 & 3.9 \pm 0.8(53 \% *)\end{array}\right] P<.01$

Data presented as mean minutes \pm SD. Construction time: insertion, sealing, positioning laser catheter, vacuum suction, lasering and closure of distal end of the graft (mounting time not included). Sealing time: time needed for circumferential anastomosis sealing. *Sealing time as a percentage of construction time.
Common objections to the ELANA technique are related to a relatively large BENIS. The nonocclusive character of the ELANA anastomotic technique and inherent lasering

TABLE 2. Operative data

\begin{tabular}{lcccc}
\hline & $\begin{array}{c}\text { Anastomosis } \\
(\mathbf{n})\end{array}$ & $\begin{array}{c}\text { Flap retrieval } \\
\text { rate (\%) }\end{array}$ & $\begin{array}{c}\text { Complete } \\
\text { hemostasis }(\%) *\end{array}$ & $\begin{array}{c}\text { Burst } \\
(\%) \dagger\end{array}$ \\
\hline Total & 40 & 83 & 95 & 0 \\
BioGlue & 20 & 85 & 100 & 0 \\
TachoSil & 20 & 80 & 89 & 0 \\
\hline
\end{tabular}

*Of the 40 anastomoses, 3 were excluded from the hemostasis assessment because of vessel wall lesions after incorrect lasering. $\dagger$ Of 20 anastomoses, 3 were excluded for burst pressure testing because of leakage through a side branch of the donor vessel. 


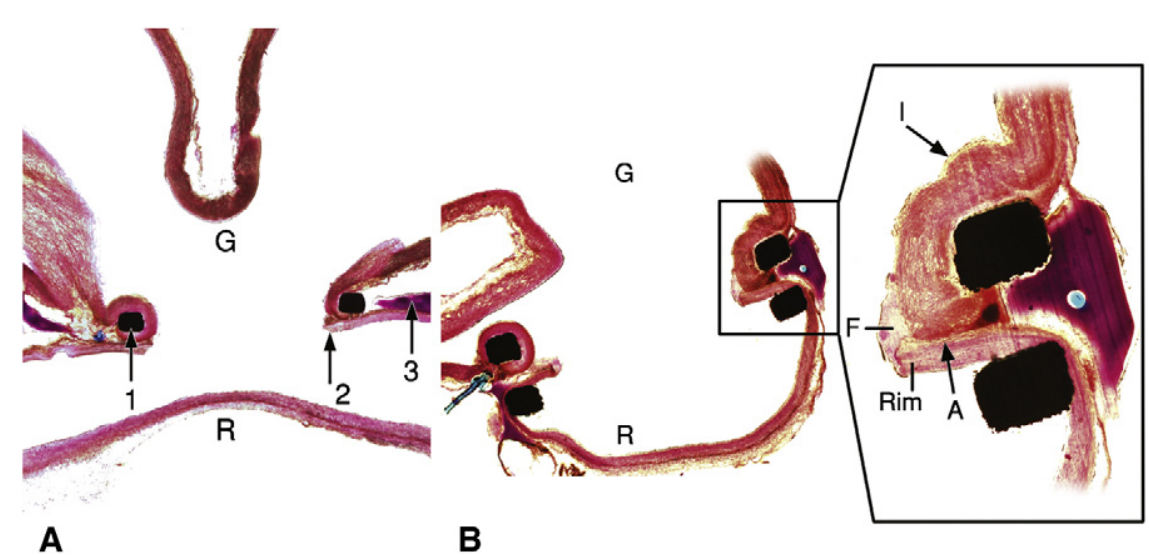

FIGURE 3. Histologic sections of the Excimer laser-assisted nonocclusive anastomosis in the rabbit abdominal aortic bypass model. A, Longitudinal section at the middle of the anastomosis (12.5 $\times$ magnification). The ring of the anastomotic connector is visible (1). A small protruding rim (2) of the abdominal aorta $(R)$ is exposed to blood. No sealant (3) is in contact with the blood. $G$, lumen of the graft. B, Transverse section (12.5× magnification). The magnified $(40 \times)$ subsection demonstrates the intima $(I)$-adventitia $(A)$ vessel wall apposition and sharp laser-cut edge (Rim) with a streamlined cover of platelets and fibrin $(F)$. $G$, Graft; $R$, recipient artery.

of the recipient artery wall (ie, unconventional intima-adventitia apposition) caused a relatively large BENIS (inherent to the distal anastomotic connectors ${ }^{2-6}$ ) of 4.4 $\mathrm{mm}^{2}$ compared with $1.3 \mathrm{~mm}^{2}$ in conventional hand-sewn anastomoses. $^{2}$ However, Buijsrogge and colleagues ${ }^{7}$ demonstrated that this unconventional intima-adventitia vessel wall apposition did not adversely affect the early patency rates in a pig coronary bypass model, even under prothrombotic low base flow conditions. Furthermore, healing of the unconventional apposition in the ELANA anastomosis has been extensively investigated using histologic and scanning electron microscopic analyses in a pig carotid bypass model, which demonstrated complete re-endothelialization of the intravascular pins and adventitial rim after 21 days, without a detriment on patency. ${ }^{8}$ However, the long-term effects of the specific characteristics (ie, a large BENIS) of the new ELANA anastomotic technique must be investigated in an off-pump porcine coronary artery bypass survival study.

\section{Potential Key Features of the ELANA Anastomotic Connector to Facilitate Distal Coronary Anastomosis}

Despite the imperfections of the studied prototype, the ELANA connector includes several interesting features compared with other coronary anastomotic connectors ${ }^{2-6}$ to facilitate distal coronary anastomosis construction. First, it is completely nonocclusive. Thus, the technique's first action is apposition of the graft onto the recipient artery before the lasered arteriotomy, without cardiac ischemia during construction. Consequently, it is possible to check for proper positioning of the connector in a bloodless field, without a time constraint. In the case of malposition, it is feasible to "bail out" and reposition the connector. Second, the laser catheter creates a rounded, 2-mm anastomotic orifice in a standardized and reproducible fashion. Finally, the anastomotic construction is simple, fast, and safe without the use of bulky applicators. Hence, it might be applicable for limited-access coronary artery bypass grafting.

\section{Procedural Limitations}

However, the current ELANA prototype connector has limitations. Essential improvements must be implemented for the technique to be successful in minimally invasive coronary bypass surgery.

First, with the current prototype, it is necessary to seal the anastomosis with a sealant to obtain complete hemostasis. This procedural step increases the number of surgical maneuvers (ie, increases surgical dexterity requirements). Moreover, the potential risk of intraluminal introduction of the sealant is a major drawback to its application. Second, because most coronary bypasses are constructed within a coronary diameter range of 1.5 to $2.5 \mathrm{~mm}$ OD, downsizing the anastomotic connector is of paramount importance. Third, the achieved flap retrieval rate in the present study $(83 \%)$ might be lower than our experience in earlier studies using the same prototype connector. ${ }^{8}$ In contrast to previous studies, ${ }^{8}$ the connector was used for sideto-side anastomotic configurations. In the present study, the side-to-side anastomotic configuration, combined with the bulkiness of the polymerized sealant, hampered perpendicular laser catheter positioning, introducing errors. Fourth, in the present design, mounting of the graft requires stitching, which is time-consuming and increases the dexterity requirements.

\section{Future Perspectives}

To address these limitations, a new, downsizable, prototype is designed. This new modified ELANA-based 
connector actively compresses the intravascular fork (ie, pins) and extravascular ring of the connector by a spring at the back of the device, resulting in an immediate leak-free anastomosis. With this design change, sealant will be redundant, simplifying and accelerating anastomotic construction. In addition, in the case of incomplete flap retrieval, it offers the potential for "bail out" (ie, retrieval of the flap manually).

\section{CONCLUSIONS}

The results of the present study have demonstrated that the ELANA-based prototype anastomotic connector is safe and feasible and can be efficiently applied in an acute rabbit abdominal aortic bypass model. Provided the limitations can be addressed, this easy-to-use and nonocclusive technique has the potential for minimally invasive coronary bypass surgery.

We acknowledge the constructive contributions of Saskia Redegeld, Glenn Bronkers, Sander van Thoor, Rik Mansvelt Beck, André van Dieren, and colleagues from the Utrecht University Central Animal Facilities.

\section{References}

1. van Doormaal TP, van der Zwan A, Verweij BH, Regli L, Tulleken CA. Gian aneurysm clipping under protection of an Excimer laser-assisted non-occlusive anastomosis bypass. Neurosurgery. 2010;66:439-47.

2. Scheltes JS, van Andel CJ, Pistecky PV, Borst C. Coronary anastomotic devices: blood-exposed non-intimal surface and coronary wall stress. J Thorac Cardiovasc Surg. 2003;126:191-9.

3. Suyker WJ, Buijsrogge MP, Suyker PT, Verlaan CW, Borst C, Grundeman PF. Stapled coronary anastomosis with minimal intraluminal artifact: the S2 Anastomotic System in the off-pump porcine model. J Thorac Cardiovasc Surg. 2004;127: 498-503.

4. Matschke KE, Gummert JF, Demertzis S, Kappert U, Anssar MB, Siclari F, et al The Cardica C-Port System: clinical and angiographic evaluation of a new device for automated, compliant distal anastomoses in coronary artery bypass grafting surgery - a multicenter prospective clinical trial. J Thorac Cardiovasc Surg. 2005; $130: 1645-52$.

5. Carrel T, Englberger L, Keller D, Windecker S, Meier B, Eckstein F. Clinical and angiographic results after mechanical connection for distal anastomosis in coronary surgery. $J$ Thorac Cardiovasc Surg. 2004;127:1632-40.

6. Filsoufi F, Farivar RS, Aklog L, Anderson CA, Chen RH, Lichtenstein S, et al Automated distal coronary bypass with a novel magnetic coupler (MVP system). J Thorac Cardiovasc Surg. 2004;127:185-92.

7. Buijsrogge MP, Grundeman PF, Verlaan CW, Borst C. Unconventional vessel wall apposition in off-pump porcine coronary artery bypass grafting: low versus high graft flow. J Thorac Cardiovasc Surg. 2002;123:341-7.

8. van Doormaal TP, van der Zwan A, Redegeld S, Verweij BH, Tulleken CA, Regli L. Patency, flow, and endothelialization of the sutureless Excimer laser assisted non-occlusive anastomosis (ELANA) technique in a pig model. $J$ Neurosurg. 2011;115:1221-30. 